\title{
The Tunka-133 EAS Cherenkov light array: Status of 2011
}

\author{
S.F. Berezhnev ${ }^{a}$, D. Besson ${ }^{\text {h }}$, N.M. Budnev ${ }^{b}$, A. Chiavassa $^{\text {e,f }}$, O.A. Chvalaev ${ }^{\mathrm{b}}$, O.A. Gress ${ }^{\mathrm{b}}$, A.N. Dyachok $^{\mathrm{b}}$, \\ S.N. Epimakhov ${ }^{a}$, A. Haungs ${ }^{i}$, N.I. Karpov ${ }^{a}$, N.N. Kalmykov ${ }^{a}$, E.N. Konstantinov ${ }^{b}$, A.V. Korobchenko ${ }^{b}$, \\ E.E. Korosteleva ${ }^{a}$, V.A. Kozhin ${ }^{\text {a }}$, L.A. Kuzmichev ${ }^{\text {a,* }}$, B.K. Lubsandorzhiev ${ }^{c}$, N.B. Lubsandorzhiev ${ }^{c}$, \\ R.R. Mirgazov ${ }^{\text {, }}$, M.I. Panasyuk ${ }^{a}$, L.V. Pankov ${ }^{b}$, E.G. Popova ${ }^{a}$, V.V. Prosin ${ }^{\text {a }}$, V.S. Ptuskin ${ }^{\text {d }}$, Yu.A. Semeney ${ }^{b}$, \\ B.A. Shaibonov Jr. ${ }^{c}$, A.A. Silaev ${ }^{\text {a }}$, A.A. Silaev Jr. ${ }^{a}$, A.V. Skurikhin ${ }^{\text {a }}$, J. Snyder ${ }^{\text {h }}$, C. Spiering ${ }^{g}$, F.G. Schröder ${ }^{\text {i }}$, \\ M. Stockham ${ }^{\text {h }}$, L.G. Sveshnikova ${ }^{\text {, }}$ R. Wischnewski ${ }^{\text {, }}$ I.V. Yashin ${ }^{\text {a }}$, A.V. Zagorodnikov ${ }^{b}$
}

a Skobeltsyn Institute of Nuclear Physics MSU, Moscow, Russia

${ }^{\mathrm{b}}$ Institute of Applied Physics ISU, Irkutsk, Russia

' Institute for Nuclear Research of RAS, Moscow, Russia

d IZMIRAN, Troitsk, Moscow Region, Russia

e Dipartimento di Fisica Generale, Universiteta di Torino, Torino, Italy

f INFN, Torino, Italy

${ }^{\mathrm{g}}$ DESY, Zeuthen, Germany

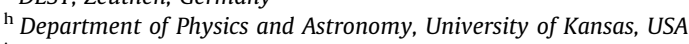

${ }^{\mathrm{i}}$ Karlsruhe Institute of Technology, Institut für Kernphysik, Karlsruhe, Germany

\section{A R T I C L E I N F O}

\section{Available online 30 December 2011}

Keywords:

EAS Cherenkov light array

Cosmic rays

Energy spectrum

Mass composition

\begin{abstract}
A B S T R A C T
A new EAS Cherenkov light array, Tunka-133, with $\sim 1 \mathrm{~km}^{2}$ geometrical area has been installed at the Tunka Valley (50 km from Lake Baikal) in 2009. The array permits a detailed study of cosmic ray energy spectrum and mass composition in the energy range $10^{16}-10^{18} \mathrm{eV}$ with a uniform method. We describe the array construction, DAQ and methods of the array calibration. The method of energy reconstruction and absolute calibration of measurements are discussed. The analysis of spatial and time structure of EAS Cherenkov light allows to estimate the depth of the EAS maximum $X_{\max }$.

The results on the all particles energy spectrum and the mean depth of the EAS maximum $X_{\max }$ vs. primary energy derived from the data of two winter seasons (2009-2011) are presented. Preliminary results of joint operation of the Cherenkov array with antennas for the detection of EAS radio signals are shown. Plans for future upgrades - deployment of remote clusters, radioantennas and a scintillator detector network and a prototype of the HiSCORE gamma-telescope - are discussed.
\end{abstract}

(c) 2012 Elsevier B.V. All rights reserved.

\section{Introduction}

The study of primary energy spectrum and mass composition in the energy range $10^{15}-10^{18} \mathrm{eV}$ is of crucial importance for the understanding of the cosmic rays origin and propagation in the Galaxy.

To measure the primary cosmic ray energy spectrum and mass composition in the mentioned energy range, the new array Tunka-133 [1,2], with nearly $1 \mathrm{~km}^{2}$ geometrical area has been deployed in the Tunka Valley, Siberia. It records EAS Cherenkov light using the atmosphere of the Earth as a huge calorimeter and has much better energy resolution $(\sim 15 \%)$ than EAS arrays detecting only charged particles.

\footnotetext{
* Corresponding author. Tel.: +7 495 9391072; fax: +7 4959393553.

E-mail address: kuz@dec1.sinp.msu.ru (L.A. Kuzmichev).
}

\section{Tunka-133}

The Tunka-133 array consists of 133 wide-angle optical detectors based on the PMT EMI 9350 with a hemispherical photocathode of $20 \mathrm{~cm}$ diameter. The detectors are grouped into 19 clusters, each cluster with seven detectors-six hexagonally arranged detectors and one in the center. The distance between the detectors inside the cluster is $85 \mathrm{~m}$.

The Cherenkov light pulse is sent via 95 m coaxial cable RG58 to the center of a cluster and digitized. The dynamic range of the amplitude measurement is about $3 \times 10^{4}$. This is achieved by means of two channels for each detector extracting the signals from the anode and from an intermediate dynode of the PMT with different additional amplification factors.

The cluster electronics includes the cluster controller, four 4-channel FADC boards, an adapter unit for connection with optical detectors and a special temperature controller. The 12 bits and 
$200 \mathrm{MHz}$ sampling FADC board is based on AD9430 fast ADCs and FPGA XILINX Spartan XC3S300 microchips. The cluster controller consists of an optical transceiver, a synchronization module, a local time clock and a trigger module. The optical transceiver operating at $1000 \mathrm{MHz}$ is responsible for data transmission and formation of $100 \mathrm{MHz}$ synchronization signals for the cluster clocks. The cluster trigger (the local trigger) is formed by the coincidence of at least three pulses from the optical detectors exceeding the threshold within a time window of $0.5 \mu \mathrm{s}$. The time mark of the local trigger is fixed by the cluster clock. The accuracy of the time synchronization between different clusters is about $10 \mathrm{~ns}$. Each cluster electronics is connected with the DAQ center by a multi-wire cable containing four copper wires and four optical fibers.

The central DAQ station consists of four DAQ boards synchronized by a single $100 \mathrm{MHz}$ generator. The boards are connected with the master PC by $100 \mathrm{MHz}$ Ethernet lines.

\section{Data processing and reconstruction of the EAS parameters}

\subsection{Data processing}

The primary data record for each Cherenkov light detector contains 1024 amplitude values with step of 5 ns (Fig. 1). Thus, each pulse waveform is recorded together with the preceding noise as a trace of $5 \mu \mathrm{s}$. To derive the three main parameters of the pulse: front delay at a level 0.25 of the maximum amplitude $t_{i}$, pulse area $Q_{i}$ and full width at half-maximum (FWHM) $\tau_{i}$, each pulse is fitted with a special self-designed smoothing curve [3].

The reconstruction of the EAS core position is performed with two methods-by fitting the measured charges $Q_{i}$ with the lateral distribution function (LDF) [4] and by fitting the measured pulse widths $\tau_{i}$ by the width-distance function (WDF) [5].

\subsection{Energy reconstruction}

As a measure of energy we used the Cherenkov light flux density at a core distance of $175 \mathrm{~m}-Q_{175}$. Connection between
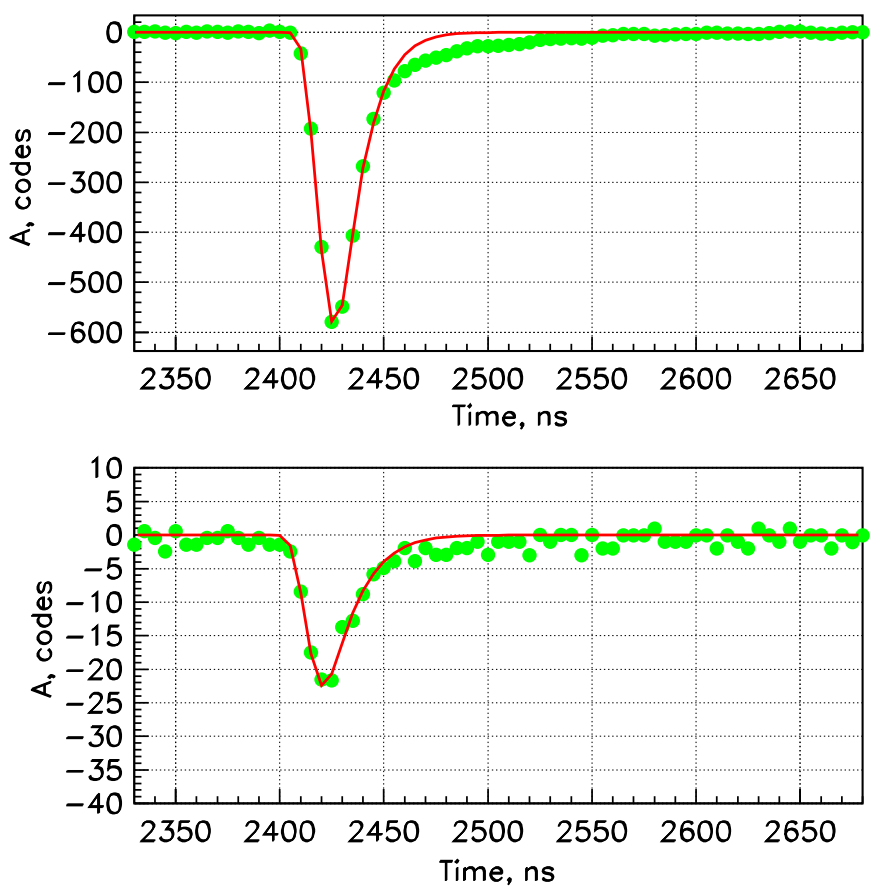

Fig. 1. Example of a pulse from one Tunka-133 detector, recorded by high and low gain channels. the EAS energy $E_{0}$ and $Q_{175}$ may be expressed by the following formula:

$E_{0}=C \cdot Q_{175}^{g}$.

It was found from CORSIKA simulation that for the energy range of $10^{16}-10^{17} \mathrm{eV}$ and zenith angle range $0^{\circ}-45^{\circ}$ the value of index $g$ is 0.95 for pure proton composition and 0.91 for pure iron composition. For energy reconstruction the value of $g$ equal to 0.93 was chosen.

To reconstruct the EAS energy from Cherenkov light flux one needs to know an absolute sensitivity of Cherenkov detectors and the atmosphere transparency. To avoid these problems, the method of normalization of the integral energy spectrum to the reference one is used. The reference energy spectrum was obtained at the QUEST experiment [6]. In that experiment, five wide-angle Cherenkov light detectors were installed at GranSasso for common operation with the EAS-TOP array. The analysis of CORSIKA simulations shows a strict correlation between the size/energy $\left(N_{e} / E_{0}\right)$ ratio and the steepness $(P)$ of the EAS Cherenkov light lateral distribution.

The relation between $N_{e} / E_{0}$ and $P$ (Fig. 2) is independent from both the mass of primary particle and the hadronic interaction model used for the simulation and provides the primary energy from the measurement of $N_{e}$ and $P$. To reconstruct the LDF steepness $P$, the knowledge of PMT absolute sensitivity and the atmosphere transparency is not needed. The integral energy spectrum of cosmic rays obtained in the QUEST experiment is used as the reference one now. The integral energy spectra obtained for each night of Tunka-133 operation is normalized to the reference one.

\subsection{Reconstruction of $X_{\max }$}

Recording the pulse waveform for each detector allows to use two methods of $X_{\max }$ reconstruction, which were developed for our experiment. The first is based on the shape of the LDF and called $P$-method. The second method, the $W$-method, is based on an analysis of time width of the Cherenkov pulses.

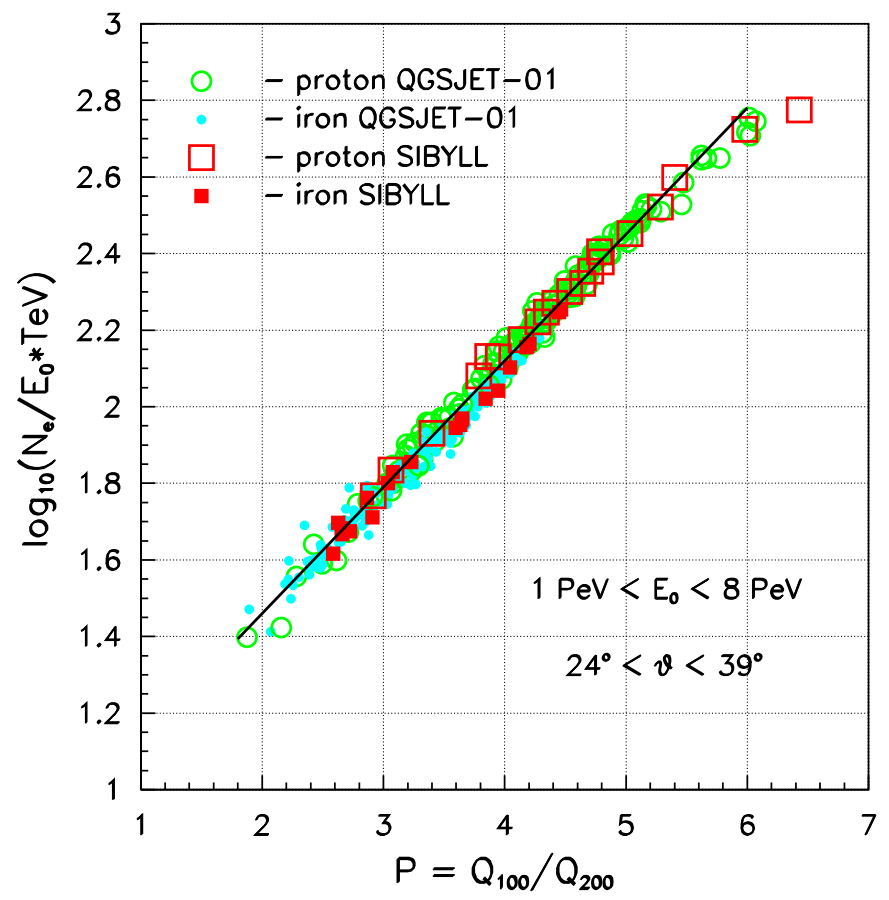

Fig. 2. $N_{e} / E_{0}$ vs. $P$. 
The LDF shape is described by an expression with a single parameter, the steepness $P$ [7]. $P$ is strictly connected with the distance from the core position to the EAS maximum [8]:

$H_{\max }=A-B \cdot P$.

MC simulations show that this relation does not depend on energy, zenith angle of the showers, mass composition and the model of nuclear interaction used for the simulation. To get a uniform estimation for $P$ over a wide range of energies, we remove from the analysis the detectors at core distances more than $200 \mathrm{~m}$ during the last step of parameter reconstruction.

The $W$-method uses the sensitivity of the pulse width at some fixed core distance to the position of the EAS maximum. We fixed this distance to $400 \mathrm{~m}$ and recalculated the values measured with detectors between 200 and $400 \mathrm{~m}$ away from the core to this distance. To characterize the pulse duration, we use the effective pulse with $\tau$

$\tau=S /\left(1.24 \cdot A_{\max }\right)$

where $S$ is the area under the pulse in dig.counts ns, $A_{\max }$ in dig.counts is its maximum amplitude.

To recalculate the pulse width to $400 \mathrm{~m}$, the width-distance function (WDF) is used. This function was constructed on the basis of CORSIKA simulation and described in Ref. [5]. It was also shown [5] that the value of $\tau(400)$ is connected with the thickness of the atmosphere between the detector and $X_{\max }\left(\Delta X_{\max }=X_{0} /\right.$ $\left.\cos \theta-X_{\max }\right)$ by the expression:

$\Delta X_{\max }=C-D \cdot \log \tau_{400}$

This relation is correct for any primary nucleus, any energy and zenith angle of the shower and any interaction model, as in the case of LDF steepness mentioned above.

\section{Energy spectrum}

\subsection{Energy spectrum for inside events}

The data taking by the Tunka-133 array started in October 2009 and has been continued during two winter seasons of 2009-2010 and 2010-2011. As a result, the data was collected for $597 \mathrm{~h}$ of 102 clean moonless nights. Only the nights with more than $2 \mathrm{~h}$ of clean weather were taken into account. The average trigger rate was about $2 \mathrm{~Hz}$. The number of recorded events was about $4 \times 10^{6}$.

For construction of the energy spectrum the events with core position inside a circle of radius $R<450 \mathrm{~m}$ from the center of the array were selected. The EAS zenith angle for the events collected into the spectrum is limited by $45^{\circ}$. The threshold energy of $100 \%$ registration efficiently for chosen area and zenith angles is $6 \times 10^{15} \mathrm{eV}$. The energy spectra obtained for two different zenith angular ranges $\left(0^{\circ}-30^{\circ}\right.$ and $\left.30^{\circ}-45^{\circ}\right)$ are in good agreement for energy more than $6 \times 10^{15} \mathrm{eV}$ (Fig. 3). It was found that the energy spectrum obtained for the first season of the array operation (2009-2010) is in good agreement with the energy spectrum from the second season (2010-2011) (Fig. 4) up to the energy $6 \times 10^{16} \mathrm{eV}$. The difference in intensity at higher energies seems to be only due to statistical fluctuation.

The indication of a "bump", found in the first season at the energy $8 \times 10^{16} \mathrm{eV}$ [9], is not seen for the second season spectrum and practically disappears in the combined spectrum (Fig. 5).

The combined energy spectrum for two seasons of the array operation is presented in Fig. 5. It contains $\sim 40,000$ events with energy $E_{0}>10^{16} \mathrm{eV}$ and $\sim 400$ events with $E_{0}>10^{17} \mathrm{eV}$. The energy spectrum of Tunka-133 is compared with that of Tunka25 [6], the predecessor of Tunka-133, in this figure. The energy spectrum beyond the knee looks rather complicated. One can see that the spectrum can be fitted by power laws with three different

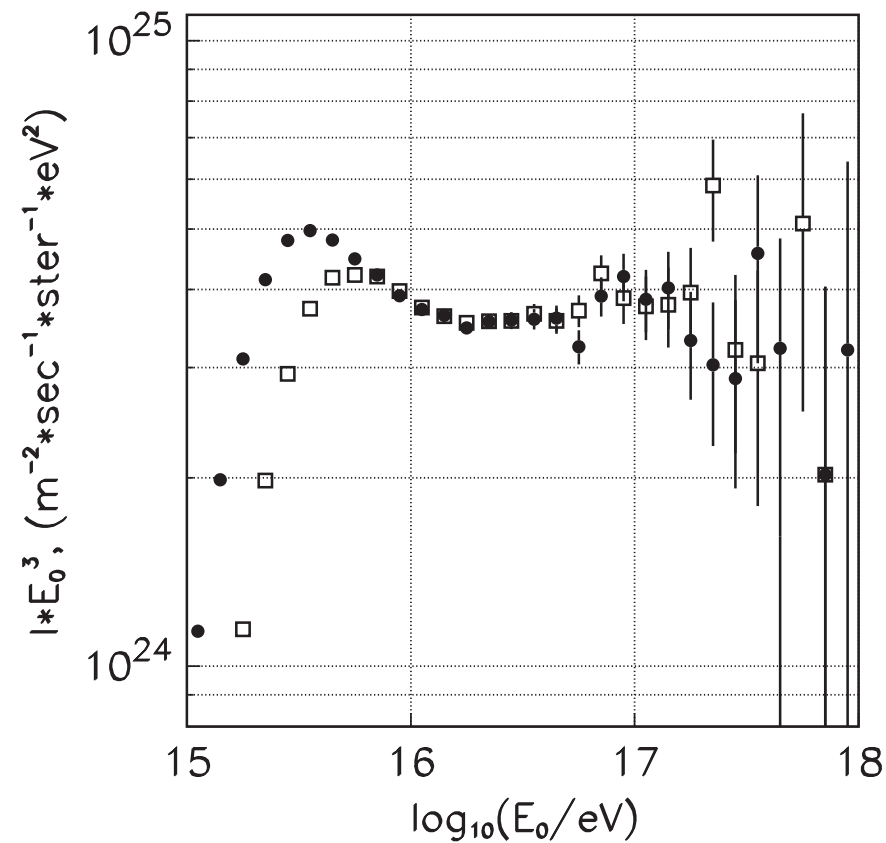

Fig. 3. Energy spectrum for two zenith angle intervals. Circles, $0^{\circ}<\theta<30^{\circ}$; squares, $30^{\circ}<\theta<45^{\circ}$.

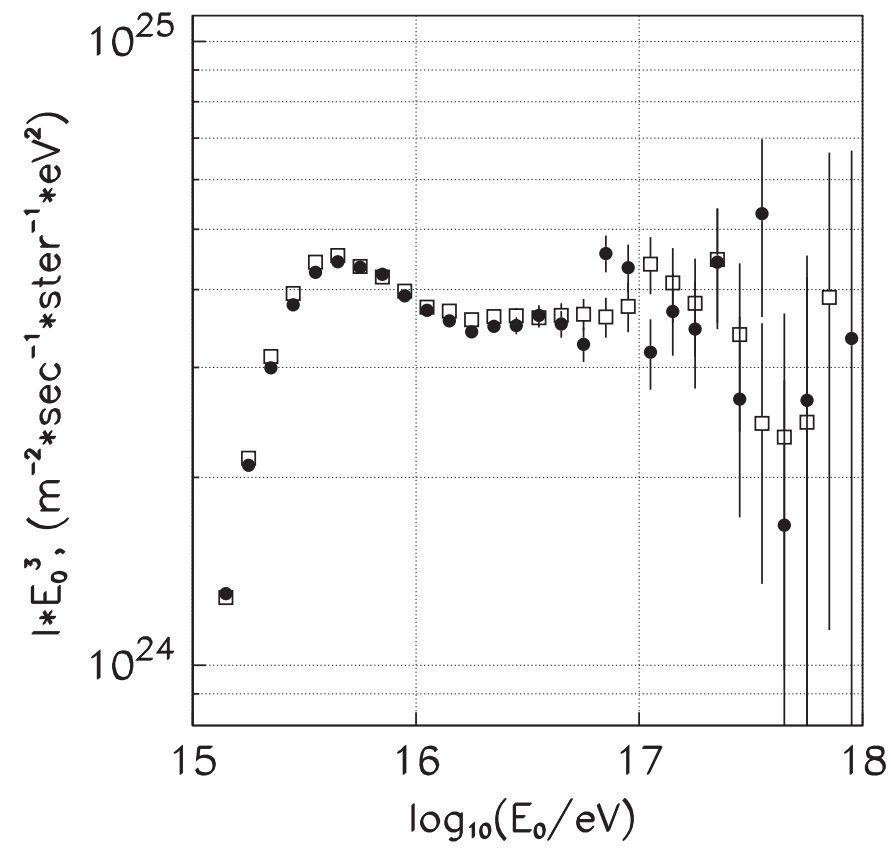

Fig. 4. Different energy spectra for two seasons of the array operation. Circles 2009-2010; squares, 2010-2011.

power law indexes: $3.21 \pm(0.01)^{\text {stat }} \pm(0.05)^{\text {syst }}$ for $6 \times 10^{15}-2 \times$ $10^{16} \mathrm{eV}, 2.95+(0.01)^{\text {stat }}+(0.05)^{\text {syst }}$ for $2 \times 10^{16}-10^{17} \mathrm{eV}$, and $3.15 \pm(0.11)^{\text {stat }} \pm(0.05)^{\text {syst }}$ for $10^{17}-10^{18} \mathrm{eV}$.

\subsection{Energy spectrum for outside events}

It was found that the energy spectrum including the outside events can be reconstructed too and this spectrum is in good agreement with the energy spectrum for inside events beyond some energy threshold. Fig. 6 presents a comparison of the spectrum for inside events $(R<450 \mathrm{~m})$ with spectra for events with core inside circles $R<550 \mathrm{~m}, R<650 \mathrm{~m}$ and $R<800 \mathrm{~m}$. 


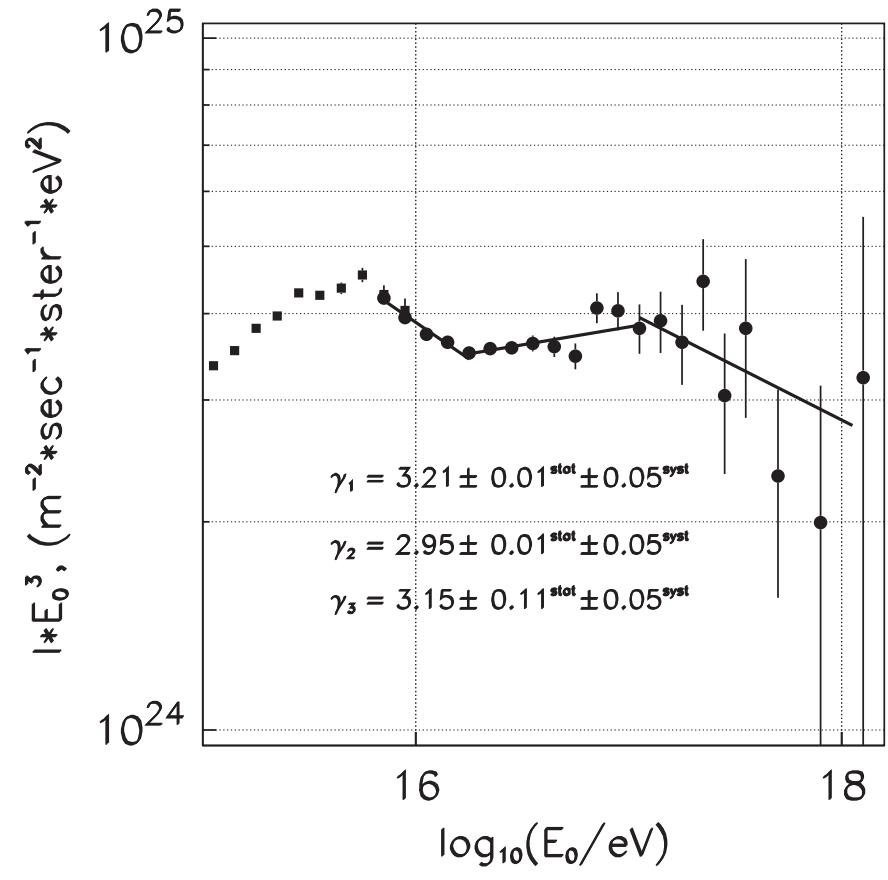

Fig. 5. Summary energy spectrum for two seasons of Tunka-133 operation (circles) and Tunka-25 energy spectrum (squares).

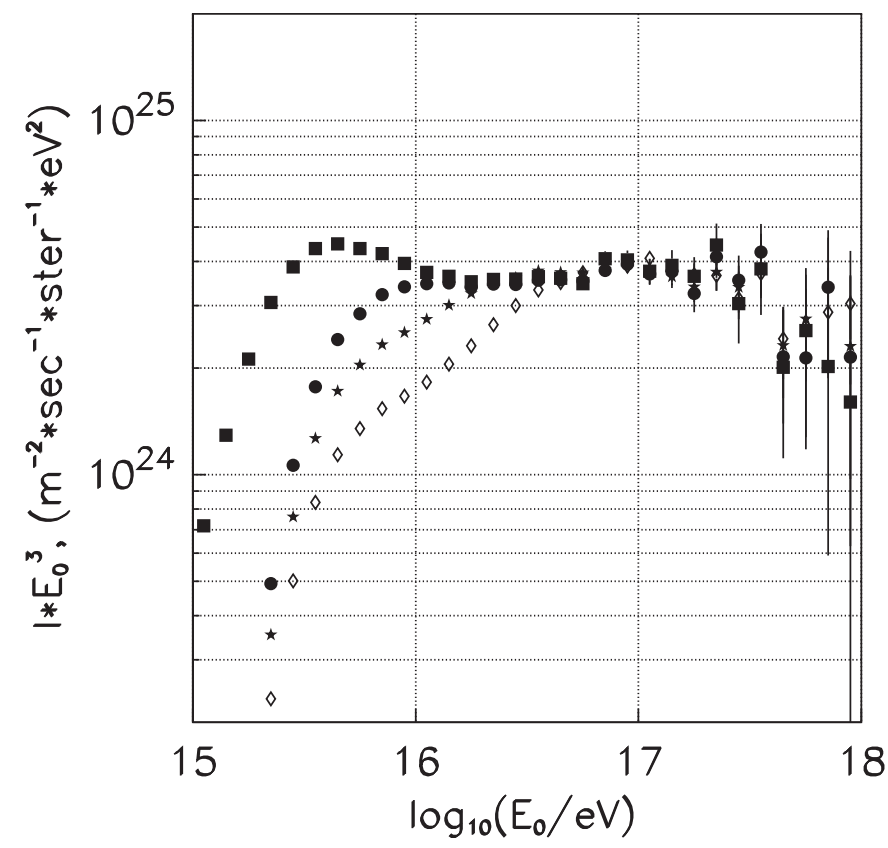

Fig. 6. Comparison of energy spectrum for inside events (squares) and spectra for outside events $R<550 \mathrm{~m}$ (circles), $R<650 \mathrm{~m}$ (stars) and $R<800 \mathrm{~m}$ (diamonds).

It is seen that the threshold energy of $100 \%$ registration efficiency increases with increasing of the radius $R$, but for $E_{0}>6 \times 10^{16} \mathrm{eV}$ all spectra are in good agreement. Based on such the result we construct the combined energy spectrum (Fig. 7) from the events with $R<450 \mathrm{~m}$ for $E_{0}<6 \times 10^{16} \mathrm{eV}$ and events with $R<800 \mathrm{~m}$ for the higher energy. This combined spectrum contains about 1200 events with $E_{0}>10^{17} \mathrm{eV}$.

The shape of the combined spectrum is nearly the same as for inside events spectrum and can be fitted by the power laws with three different indexes: $3.21 \pm(0.01)^{\text {stat }} \pm(0.05)^{\text {syst }}$ for $6 \times 10^{15}$ $-2 \times 10^{16} \mathrm{eV}, 2.93 \pm(0.01)^{\text {stat }} \pm(0.05)^{\text {syst }}$ for $2 \times 10^{16}-10^{17} \mathrm{eV}$, $3.20 \pm(0.06)^{\text {stat }} \pm(0.05)^{\text {syst }}$ for $10^{17}-10^{18} \mathrm{eV}$.

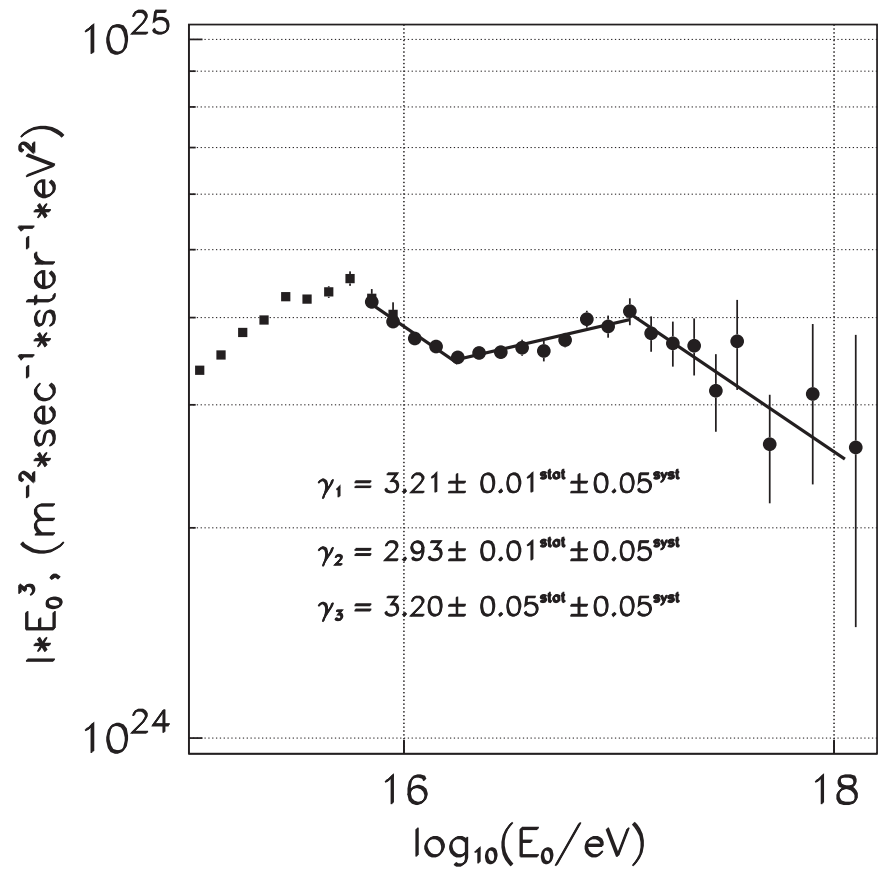

Fig. 7. Combined energy spectrum. Squares, Tunka-25 data.

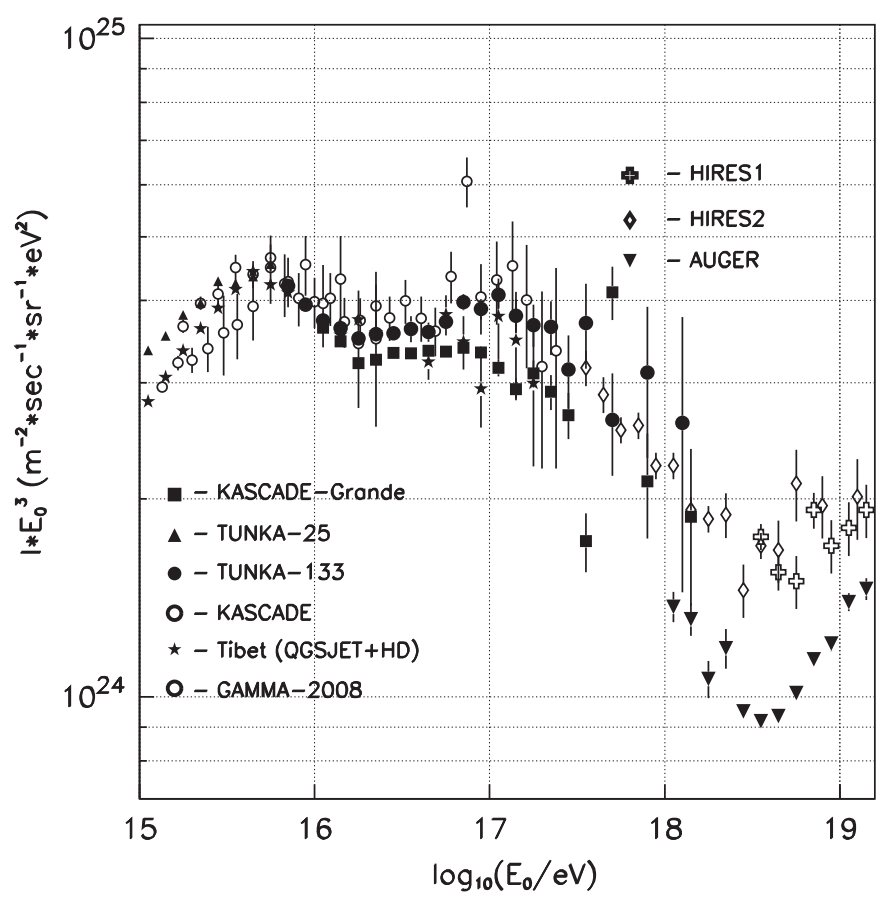

Fig. 8. Tunka-133 all particle energy spectrum in comparison with results of other experiments.

\subsection{Comparison with results of other experiments}

A comparison of the Tunka-133 spectrum with the results of other experiments is presented in Fig. 8. Our spectrum is in good agreement with spectra of KASCADE [10], Tibet [11] and GAMMA [12] arrays. The energy range covered by our spectrum $\left(10^{16}\right.$ $10^{18} \mathrm{eV}$ ) is nearly the same as covered by the KASCADE-Grande array data [13].

Both spectra reproduce the same structures: decrease of power law index at $2 \times 10^{16} \mathrm{eV}$ and an increase at $10^{17} \mathrm{eV}$. The 
difference in absolute cosmic ray flux intensity for Tunka-133 and KASCADE-Grande spectra is smaller than $30 \%$ even at $10^{17} \mathrm{eV}$ where the difference reaches the maximum value. It should be mentioned that if for $g$ (see expression (1)) a value of 0.91 is used, the difference in the intensity becomes smaller than $10 \%$.

\section{Phenomenological approach}

The parameters derived from CORSIKA simulation may slightly differ from the experimentally measured parameters. For instance, the linear relation between $P$ and $H_{\max }$ observed for MC simulation (Eq. (2)) may hold also for the experiment, but with a slightly different slope. For our recalculation procedure we used the slope derived from experimental data, not from MC, i.e. choosing a "phenomenological approach".

In our case we can use the zenith angle dependence of the parameter $P$. This experimental dependence for the fixed energy $E_{0}=10^{16} \mathrm{eV}$ is shown in Fig. 9. This mean dependence was constructed using all the 14,400 events from the energy bin $16.0<\log _{10}\left(E_{0} / \mathrm{eV}\right)<16.1$. The mean zenith angle can be recalculated to the mean distance to the EAS maximum. To make this recalculation we use the model of the atmosphere from Ref. [14] for the real experimental conditions $\langle t\rangle=-30{ }^{\circ} \mathrm{C}$ and $X_{0}=965 \mathrm{~g} \mathrm{~cm}^{-2}$. This model gives the following expression for the inclined distance to the EAS maximum in $\mathrm{km}$ :

$H_{\max }=\frac{H_{0}}{\cos \theta} \cdot\left(1-\left(\frac{X_{\max } \cdot \cos \theta}{X_{0}}\right)^{0.0739}\right)$

where $H_{0}=96 \mathrm{~km}$.

To fix the absolute value of $\left\langle H_{\max }\right\rangle$ we need to fix the mean value $\left\langle X_{\max }\right\rangle$ for the above mentioned energy. The most reliable experimental estimation of the mean depth of maximum by the data of our previous Tunka-25 experiment is $\left\langle X_{\max }\right\rangle=$ $560 \mathrm{~g} \mathrm{~cm}^{-2}$ [8]. Recalculated to the mean $\langle\ln A\rangle$, this value is in good agreement with the results of some other experiments [15]. The so-derived experimental recalculated dependence $H_{\max }$

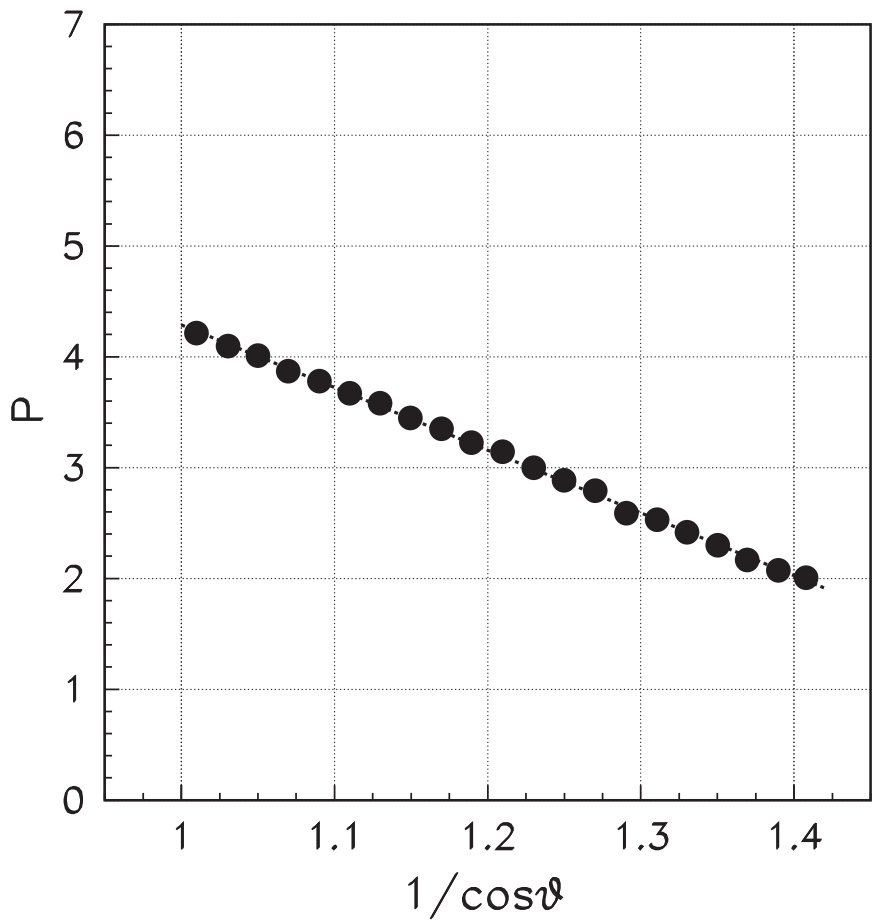

Fig. 9. $P$ vs. $\theta$ dependence from experiment. vs. $P$ is shown in Fig. 10. It can be fitted with a linear expression:

$H_{\max }=A_{1}-B_{1} \cdot P$

where $A_{1}=12.67 \mathrm{~km}$ and $B_{1}=2.09 \mathrm{~km}$.

This expression was used for the estimation of $X_{\max }$ for each individual event.

A similar approach for the second parameter sensitive to the position of EAS maximum $\tau(400)$ can be used but for higher energy because of the greater energy threshold of this method. The zenith angular dependence of $\tau_{400}$ for the logarithmic energy bin $16.5<\log _{10}\left(E_{0} / \mathrm{eV}\right)<16.6 \mathrm{eV}$ is shown in Fig. 11 . The second method normalized to the $X_{\max }$ obtained by the first one for the energy $3 \times 10^{16} \mathrm{eV}$ to fix the absolute scale of $\Delta X_{\max }$. This approach results in an expression connecting $\tau_{400}$ with the thickness of matter between the array and the EAS maximum in $\mathrm{g} \mathrm{cm}^{-2}$ :

$\Delta X_{\max }=C_{1}-D_{1} \cdot \log \tau(400)$

where $C_{1}=3493 \mathrm{~g} \mathrm{~cm}^{-2}, D_{1}=1689 \mathrm{~g} \mathrm{~cm}^{-2}$.

The result is shown in Fig. 12.

\section{Experiment: $\left\langle X_{\max }\right\rangle$ vs. $\boldsymbol{E}_{\mathbf{0}}$}

The experimental dependence of mean $\left\langle X_{\max }\right\rangle$ vs. primary energy $E_{0}$ obtained with two methods described above in the energy range $5 \times 10^{15}-3 \times 10^{17} \mathrm{eV}$ is presented in Fig. 13. The new measurements are compared with that obtained with our previous array Tunka-25 and with the theoretical curves simulated with QGSJET-01 model for primary protons and iron nuclear. The first conclusion is that the threshold of the $W$-method is higher than that of the LDF steepness method, but the experimental points obtained by both methods coincide within the statistical errors.

Much higher statistics of Tunka-133 points has led to the much smoother behavior of the experimental dependence compared with the Tunka-25 data. The experimental points go closer to the iron curve with energy grow from the knee to about $10^{17} \mathrm{eV}$. There is a tendency of a backwards movement of the experimental points to the proton curve at the energy more than $10^{17} \mathrm{eV}$, but the statistical errors are too big to insist on such conclusion.

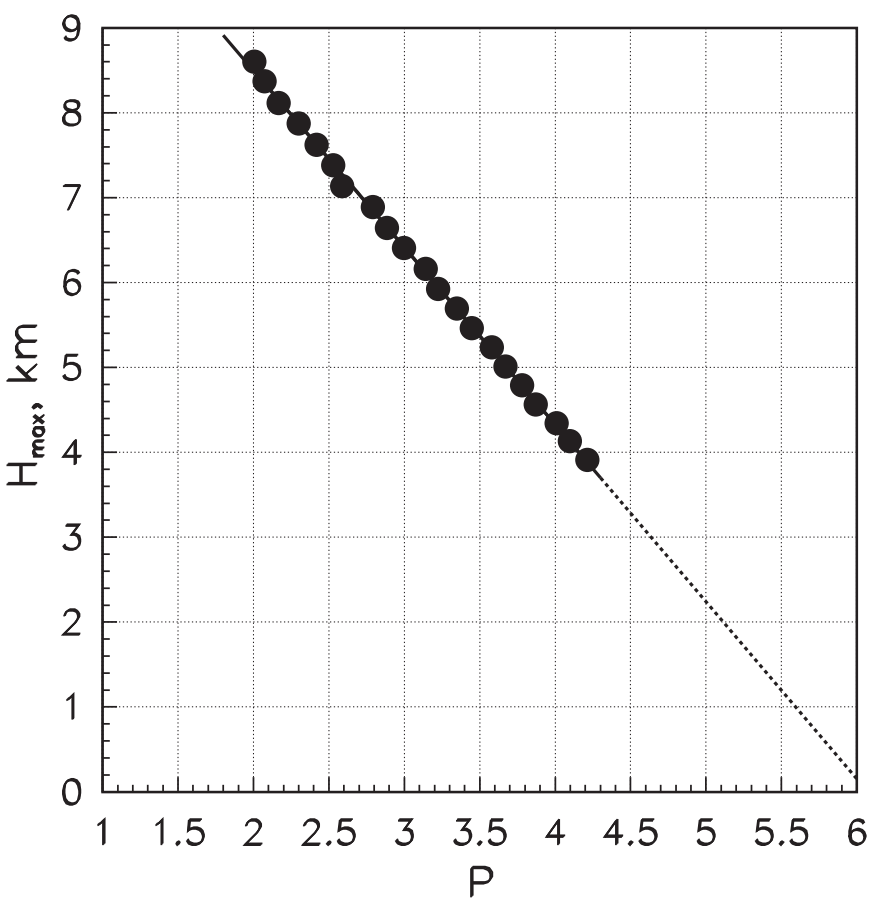

Fig. 10. $H_{\max }$ vs. $P$ dependence from experiment. 


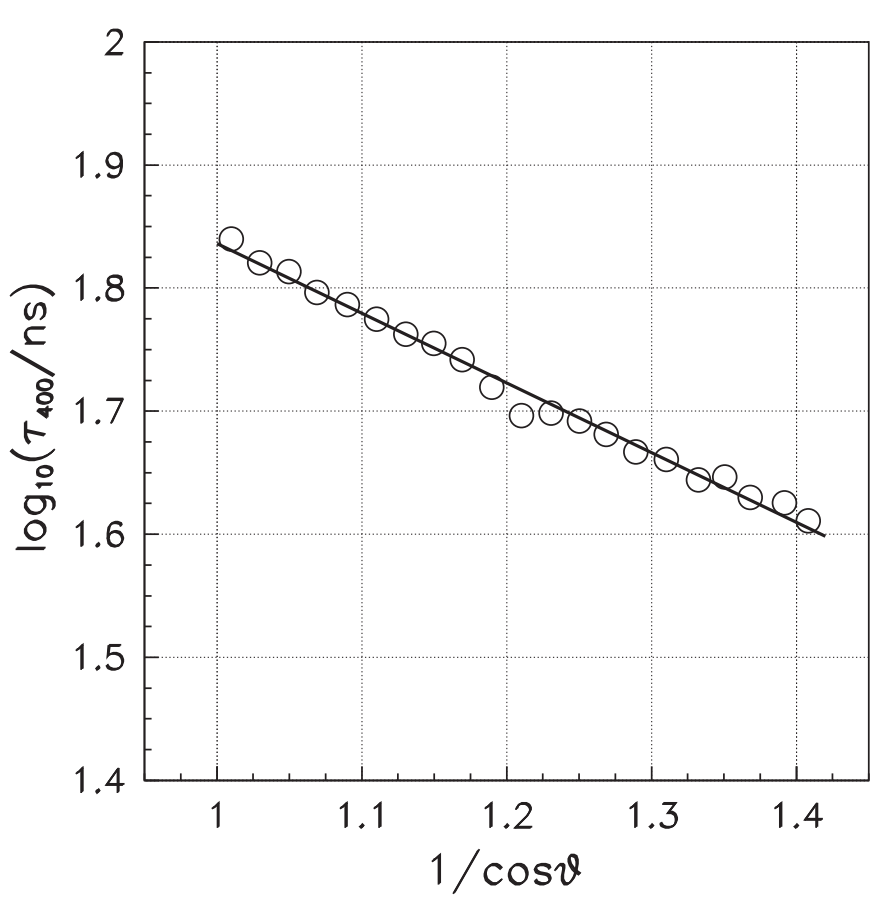

Fig. 11. $\tau_{400}$ vs. $\theta$ dependence from experiment.

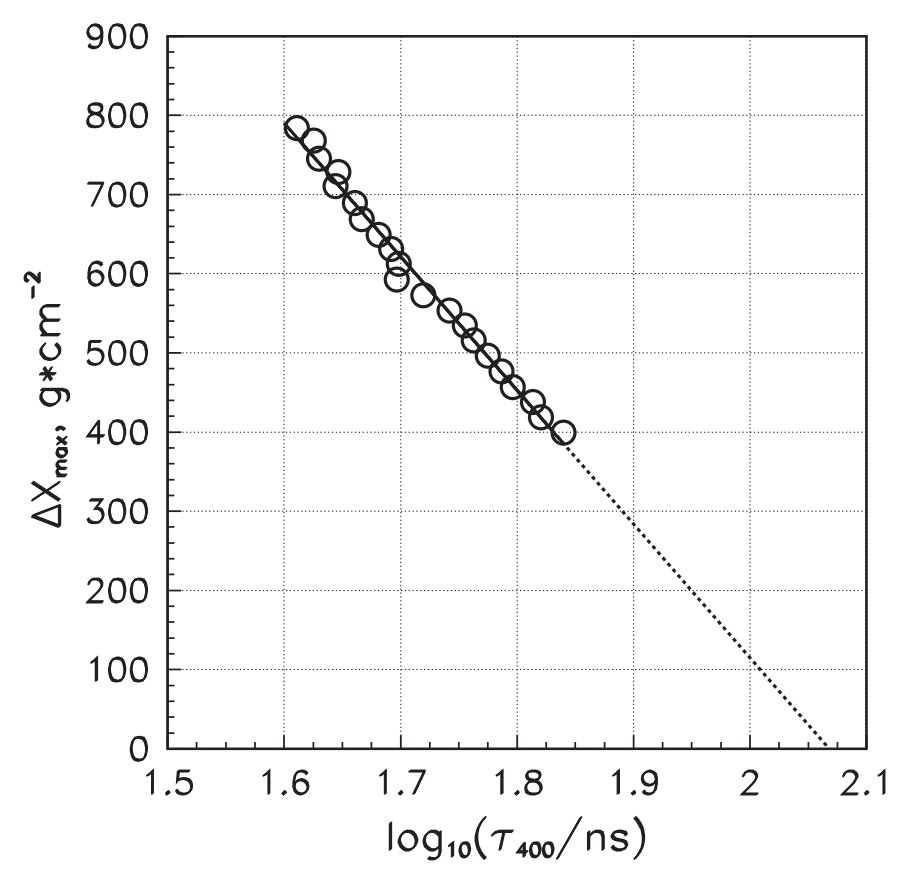

Fig. 12. $\Delta X_{\max }$ vs. $\tau(400)$ dependence from experiment.

The mean values of $\left\langle X_{\max }\right\rangle$ can be recalculated to the mean values of $\langle\ln A\rangle$ by a simple method of interpolation taking into account the corrections to the asymmetry of the $X_{\max }$ distribution, estimated at our previous work [4]. The result of such approach for the points derived from the LDF steepness analysis is shown in Fig. 14. We have to note that this procedure can give different absolute values of $\langle\ln A\rangle$ for different supposed models of nuclear interaction. The model QGSJET-01 we use for the analysis provides the highest position of the EAS maximum as compared with the other models used now for simulations. The most deep position of EAS maximum can be obtained using the QGSJET-II-03 model. The mean difference in $X_{\max }$ between these models is about $20 \mathrm{~g} \mathrm{~cm}^{-2}$. Using of this last model can increase the

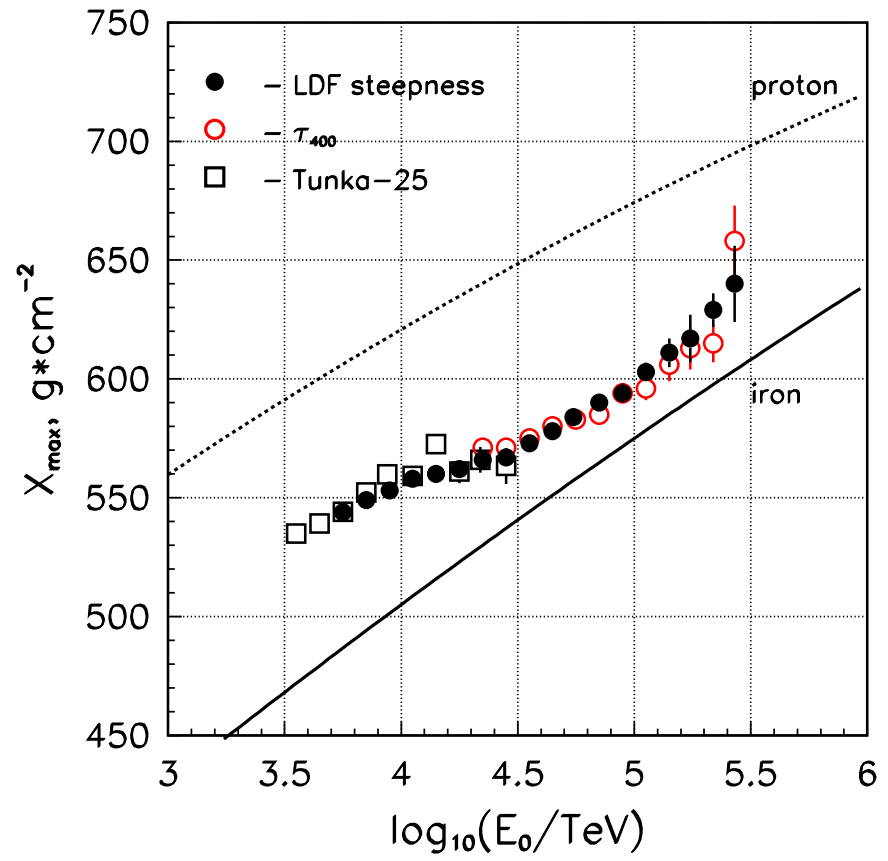

Fig. 13. Experimental $\left\langle X_{\max }\right\rangle$ vs. $E_{0}$ dependence for Tunka-133, compared with Tunka-25.

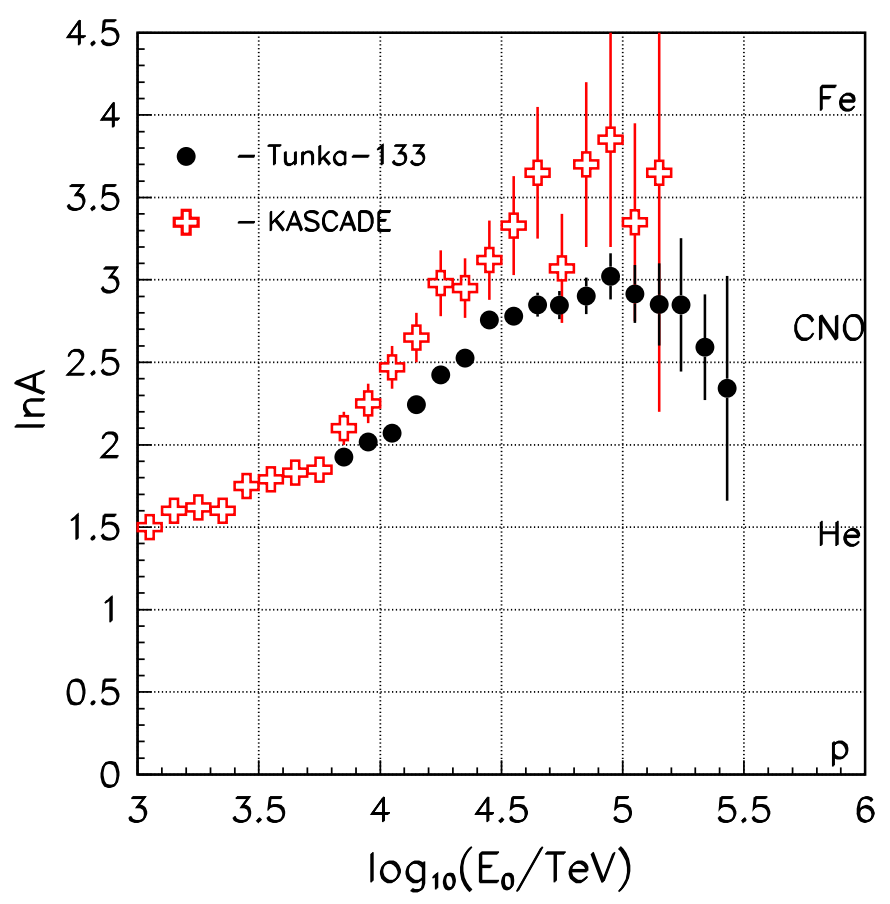

Fig. 14. Experimental $\langle\ln A\rangle$ vs. $E_{0}$ dependence.

estimation of $\langle\ln A\rangle$ to about 0.8 for the same experimental value of $\left\langle X_{\max }\right\rangle$.

The experimental points are compared with that obtained from the analysis of the muon/electron ratio in the KASCADE [15] experiment in Fig. 14.

\section{Other results}

\subsection{Measurements of radio signals from EAS}

To study whether, in addition to the Cherenkov light, the radio emission of air showers can be detected at Tunka, a SALLA 
antenna [16] was deployed at Tunka cluster 7 in summer 2009. SALLA is read out simultaneously with the PMTs of that cluster. Indeed numerous radio pulses could be detected, but most of them are due to RFI by the Tunka electronics or other background. However, for Tunka events with a high PMT signal, i.e. a high primary energy, an accumulation of events with a radio pulse shortly before the PMT pulses is observed (see Fig. 15 for an example). Selecting these events, about 70 radio candidate events have been found within the season 2009/2010.

Several tests have been performed to confirm whether the detected radio pulses are really from air showers or due to any kind of background generated by Tunka. One test was to check for which events in a selection of high quality and high energy Tunka events a radio pulse could be detected (see Fig. 16). Consistent with the results of other radio experiments (e.g., LOPES [17]), a detection is more likely in the east-west aligned antenna and for high energies. Furthermore, the ratio between the amplitude in the east-west and north-south aligned antenna has been compared to REAS3 [18] simulations. For 7 out of 11 events which show a radio pulse in both polarizations, the amplitude ratio is compatible within the measurement uncertainties estimated with a formula derived for LOPES [19]. This is another indication that at

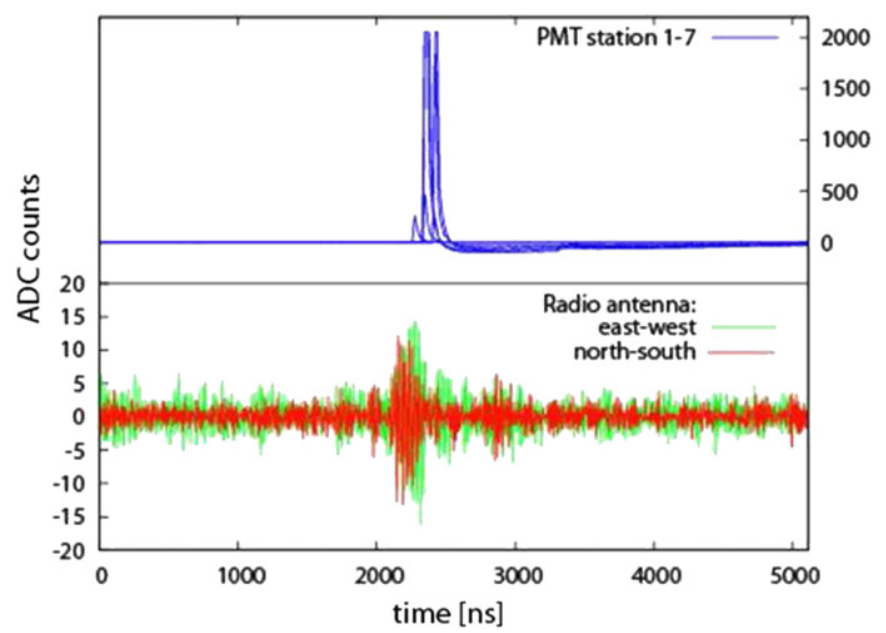

Fig. 15. One of the radio candidate events. A radio pulse is detected in both polarizations shortly before the signal of the PMTs.

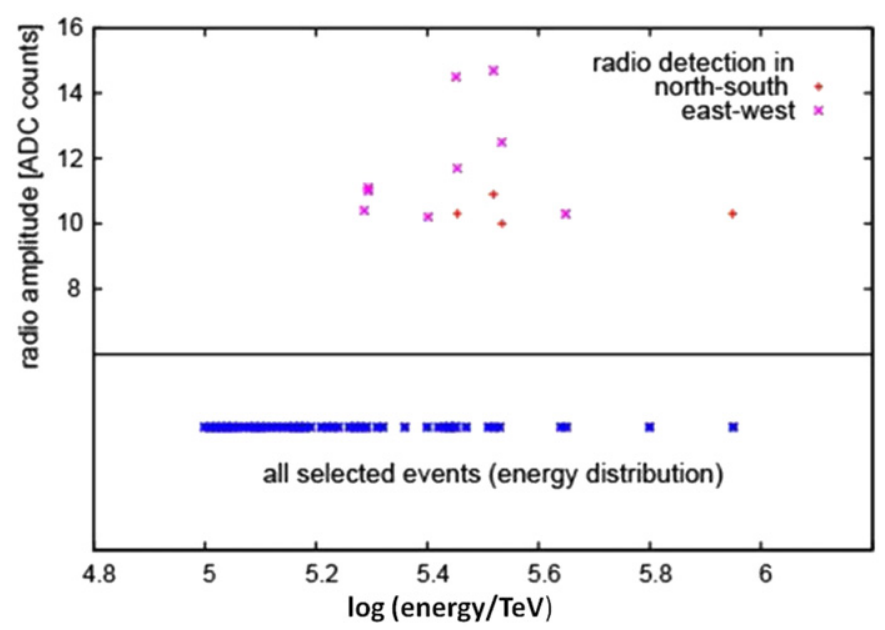

Fig. 16. Energy distribution of a selection of Tunka events and the radio amplitude for those of these events which show a radio pulse in at least one polarization: radio pulses are preferred visible for higher energies. least most of the detected radio candidates are due to the radio emission of air showers.

\subsection{Time calibration}

For time synchronization of the optical modules of the Tunka133 array, a dedicated calibration system has been developed [20]. The calibration system is based on a powerful nanosend LED light source especially designed for the system. The light source consists of four high $\ln \mathrm{GaN} / \mathrm{GaN}$ royal blue $\left(\lambda_{\max }=450 \mathrm{~nm}\right)$ LEDs with $100^{\circ}$ full emission angle. Thus, the light source covers $2 \pi$ azimuth angle.

Each LED of the source is driven by its own avalanche transistor driver and provides $\sim 10^{12}$ photons per pulse, with $\sim 4$ ns pulse width (FWHM). All LED drivers of the source are triggered simultaneously. Since the light source is installed a few meters above the ground it can, in principle, illuminate the whole array, given that all optical modules of the array are equipped with appropriate reflectors.

\section{Plans for upgrading}

The Tunka-133 facility will be significantly upgraded until fall 2013. In 2011 we will install additional clusters of optical detectors at large distance from the center of the array ("distant clusters"). In 2011-2013, we plan to install radio antennas and scintillation detectors for common operation with Tunka-133.

\subsection{Deployment of six distant clusters}

To improve the accuracy of energy reconstruction for the events with the cores located outside the array geometry we plan to install six new clusters at $1 \mathrm{~km}$ radius around the center of Tunka-133 [9]. These additional 42 optical detectors will increase the effective area for $E_{0}>10^{17} \mathrm{eV}$ by a factor of four. The first distant cluster was deployed in autumn 2010, the next five will be deployed during summer-autumn 2011.

\subsection{Net of radio antennas at Tunka}

The positive results of common operation of the first SALLA antenna and the Tunka-133 array suggest that it would be interesting to install a net of the same type of antennas at Tunka. The principle aim of such a radio-array is to show whether radio measurements of cosmic ray air showers allow the same precision for cosmic ray measurements as Cherenkov light measurements. In particular, the precision of the energy and mass determination of primary cosmic-ray particles will be investigated. The determination of the precision is a crucial input for the design of the next-generation large cosmic-ray observatories, since radio measurements are not limited to dark, moonless nights as other calorimetric detection techniques, like Cherenkov or fluorescence light measurements.

In autumn 2011 we plan to install additional 2-3 antennas, further $\sim 20$ antennas will be installed in 2012 .

\subsection{Scintillation detectors}

The deployment of scintillation counters within the Tunka array provides a cross-calibration of different methods of air shower parameters reconstruction.

In 2010, a test variant of a scintillation detector was installed. It can operate independently as well as triggered by Tunka-133. The mass production of detectors will start at the beginning of 2012. We plan to produce 20 scintillation detectors with $10 \mathrm{~m}^{2}$ 
area each. As alternative possibility, it is under discussion, to move scintillator counters from Karlsruhe to Tunka after the shutdown of the KASCADE-Grande array in spring 2012.

Scintillation detectors will be installed with a spacing of 150 $170 \mathrm{~m}$ and cover an area of about $1 \mathrm{~km}^{2}$. This will permit to perform a new QUEST-like experiment [6] and to obtain a point of absolute energy calibration at $3 \times 10^{16}-10^{17} \mathrm{eV}$ in $2012-2013$.

\subsection{HiSCORE station}

The aim of the HiSCORE project [21] is to explore the gammaray skymap beyond $10 \mathrm{TeV}$ with a future large-area $\left(10-100 \mathrm{~km}^{2}\right)$ wide-angle ( $>0.6 \mathrm{sr}$ ) non-imaging cosmic ray and gamma-ray air shower detector.

HiSCORE is a net of detector stations with four PMT-channels equipped with Winston cones. The station light-collecting area $\left(0.5 \mathrm{~m}^{2}\right)$ will be a factor 16 larger than that of an Tunka optical detector, resulting in a significantly lower energy threshold. The first such station will be installed in fall 2011 at the Tunka site. In 2012-2013, a net of such stations (HiSCORE Engineering Array (EA)) will be installed for common operation with Tunka-133. The aim of the HiSCORE EA is to prove methods of EAS reconstruction before deployment of a full scale array.

\section{Acknowledgments}

This work was supported by Russian Federation Ministry of Science and Education (G/C 16.518.11.7051, 14.740.11.0890, and P681), the Russian Foundation for Basic Research (Grants 10-0200222, 11-02-10005, 11-02-0409, 11-02-91332, and 11-0212138). Authors acknowledge J. Oertlin (KIT) for his help in the analysis of the radio-data.

\section{References}

[1] N.M. Budnev, et al., Tunka Collaboration, in: Proceedings of the 30th ICRC, Merida, Yucatan, Mexico, 2007, arXiv:0801.3037.

[2] N.M. Budnev, et al., Tunka Collaboration, in: Proceedings of the 31st ICRC, Lodz, Poland, vol. 1, 2009, p. 1069, arXiv:1003.0089.

[3] E.E. Korosteleva, et al., in: Proceedings of the 31st ICRC, Lodz, Poland, vol. 1, 2009, p. 0492.

[4] N.M. Budnev, et al., Tunka Collaboration, Nuclear Physics B: Proceedings Supplements 190 (2009) 247.

[5] B.V. Antokhonov, et al., Tunka Collaboration, Nuclear Physics B: Proceedings Supplements 212-213 (2011) 53.

[6] E.E. Korosteleva, et al., Nuclear Physics B: Proceedings Supplements 165 (2007) 74.

[7] EAS-TOP Collaboration, E.E. Korosteleva, L.A. Kuzmichev, V.V., Prosin, in: Proceedings of the 28th ICRC, Tsukuba, Japan, vol. 1, 2003, p. 89.

[8] N.M. Budnev, et al., Tunka Collaboration, Bulletin of the Russian Academy of Sciences: Physics 71 (4) (2007) 474

[9] B.V. Antokhonov, et al., Tunka Collaboration, in: 25th Texas Symposium on Relativistic Astrophysics, 2010, p. 138 (published in Proceedings of Science, POS, Texas 2010).

[10] M. Roth, et al., KASCADE Collaboration, in: 28th ICRC, Tsukuba, vol. 1, 2003, p. 139.

[11] M. Amenomori, et al., The Astrophysical Journal 678 (2008) 1165.

[12] A.P. Garyaka, et al., Journal of Physics G: Nuclear and Particle Physics 35 (2008) 115201.

[13] A. Haungs, For KASCADE-Grande Collaboration, in: Proceedings of the 32nd ICRC, Beijing, 2011, ID =677.

[14] B.E. Zuev, et al., Optical Weather, Science, Novosibirsk, Russia, 1990.

[15] T. Antoni, et al., KASCADE Collaboration, Astroparticle Physics 24 (2005) 1.

[16] O. Krömer, et al., LOPES Collaboration, in: Proceedings of the 31st ICRC, Łódź, Poland, 2009, ID =1232.

[17] H. Falcke, et al., LOPES Collaboration, Nature 435 (2005) 313.

[18] M. Ludwig, T. Huege, Astroparticle Physics 34 (2010) 438.

[19] F.G. Schröder, et al., LOPES Collaboration, Nuclear Instruments and Methods in Physics Research Section A 662 (11) (2012) S238-S241.

[20] B.K.Lubsandorzhiev, et al., in: Proceedings of the 32nd ICRC, Beijing, 2011, ID $=1048$.

[21] M. Tluczykont, et al., in: Proceedings of the 31st ICRC, Lodz, Poland, arXiv:astro-ph/0909.0445. 